\title{
Cautious strategy update promotes cooperation in spatial prisoner's dilemma game
}

Yongkui Liu

Lin Zhang

Xiaojie Chen (chenx@iiasa.ac.at)

Lei Ren

Long Wang

\section{Approved by}

Ulf Dieckmann

Director, Evolution and Ecology Program

June 2015

Interim Reports on work of the International Institute for Applied Systems Analysis receive only limited review. Views or opinions expressed herein do not necessarily represent those of the Institute, its National Member Organizations, or other organizations supporting the work. 


\title{
Cautious strategy update promotes cooperation in spatial prisoner's dilemma game
}

\author{
Yongkui Liu ${ }^{\mathrm{a}, \mathrm{b}}$, Lin Zhang ${ }^{\mathrm{b}}$, Xiaojie Chen ${ }^{\mathrm{c}}$, Lei Ren ${ }^{\mathrm{b}}$, \\ Long Wang ${ }^{\mathrm{d}}$ \\ ${ }^{a}$ School of Electronic and Control Engineering, Chang'an University, Xi'an \\ 710064, China \\ ${ }^{\mathrm{b}}$ School of Automation Science and Electrical Engineering, Beihang University, \\ Beijing 100191, China \\ ${ }^{\mathrm{c}}$ Evolution and Ecology Program, International Institute for Applied Systems \\ Analysis (IIASA), Schlossplatz 1, 2361 Laxenburg, Austria \\ ${ }^{\mathrm{d}}$ Center for Systems and Control, State Key Laboratory for Turbulence and \\ Complex Systems, College of Engineering, Peking University, Beijing 100871, \\ China
}

\begin{abstract}
Strategy update may involve costs or risks in evolutionary games since the new strategy may lead to a lower payoff and even a cascading failure in the future. Therefore, individuals may be cautious in the strategy updating process. Here we study the evolutionary prisoner's dilemma game on a square lattice by the Fermi updating rule, where each individual is assigned a cautiousness index that controls its learning activity. Interestingly, it is found that cooperation can be significantly promoted when individuals are cautious to update strategy. In particular, there exists an optimal range of the degree of cautiousness resulting in the highest cooperation level. The remarkable promotion of cooperation, as well as the emerging phase transition is explained by the configuration analysis. We also demonstrate the robustness of the promotion of cooperation with respect to the initial conditions consisting of different fractions of cooperators. Our study uncovers the nontrivial effect of individual cautiousness on cooperation, and thus provides an explanation of the ubiquitous cooperation from the perspective of individual characteristics.
\end{abstract}

Key words: Prisoner's dilemma, Cooperation, Cautious PACS: 02.50.Le; 87.23.Kg; 89.75.Ge

Email address: yongkuiliu@163.com (Yongkui Liu). 


\section{Introduction}

Cooperation is of great significance in both animal world and human society. The advancement, development and prosperity of human society rely on cooperation among individuals to a large extent [1,2]. Thus, exploring the underlying mechanisms favoring the evolution of cooperation can make us better understand the evolution of cooperation, and then apply them in practical social systems. Evolutionary game theory provides a powerful theoretical framework for investigating the origin of cooperation among selfish and unrelated individuals [3-5]. The fundamental contradiction in biological and social systems is the conflict of interests between individuals and their group [6]. The prisoner's dilemma game, as well as the public goods game can well characterize this tension, and thus becomes the prominent mathematical metaphors to investigate social dilemmas.

In this paper, we employ the prisoner's dilemma game which describes pairwise interactions to explore cooperative mechanisms. In this simple game, there are two available strategies, i.e., cooperation (C) and defection (D), for each of the two participating players. When playing the game, both players receive the reward $R$ for mutual cooperation, but the punishment $P$ for mutual defection $(R>P)$. In the case of a cooperator (who adopts $\mathrm{C}$ strategy) playing against a defectors (who adopts D strategy), the former receives the lowest sucker's payoff $S$ and the latter attains the highest payoff $T$ (the temptation to defect), thus $T>R>P>S$. It is also required that $2 R>T+S$, indicating that if both players cooperate, the collective income is maximized. The prisoner's dilemma game can well characterize the tension between individual and group interests: mutual cooperation guarantees the highest collective income, it is, however, challenged by the unilateral defection, which results in the highest individual payoff $T$.

Since the pioneering work of Nowak and May [7], spatial games have received much attention [8]. Initially, players are arranged on simple lattices, such as square lattices [9]. Afterwards, with the rapid development of studies of complex networks, evolutionary games are gradually transplanting into complex networks and have been extensively studied [10-19]. For spatial games, an important impact on cooperation stems from the underlying network structure, and sometimes it is determinant for the success of cooperative behavior $[10,14]$. Allowing individuals to switch their interaction partners adaptively can also ensure the evolutionary success of cooperators [20-24]. For spatial games, many other approaches facilitating the evolution of cooperation have also been considered, including the introduction of noises to payoffs and updating rules [25-29], asymmetry between interaction and replacement graphs $[30,31]$, interaction stochasticity [32], diversity [33,34], as well as differences between time scales of game dynamics [35-38]. The factors supporting the evo- 
lution of cooperation in the individual level involve memory effects [39], heterogeneous teaching activity [40-42], preferential learning [43,44], aspiration [45-47], migration [48-51], local contribution [52,53], and myopically selective interactions $[54,55]$, to name but a few examples studied in recent years. So far, overall there exist five prominent mechanisms facilitating the emergence and maintenance of cooperation, which are kin selection, direct reciprocity, indirect reciprocity, network reciprocity, and group selection [56].

Now we would like to concentrate on another intrinsic property of individualscautiousness. Cautiousness is ubiquitous in real-life society but is largely ignored in previous studies. The cautiousness of individuals is induced by the risk or cost of strategy update since strategy update may lead to a lower payoff and even a cascading failure in the future. It corresponds to the phenomenon that to avoid the risk or failure in game interactions, individuals are cautious to update strategy. For example, cautious individuals usually begin to learn their neighbors only when the payoff difference exceeds a certain threshold rather than immediate learn their neighbors as long as the neighbors perform better. As we will show, introducing the cautiousness of individuals in the strategy updating process can unexpectedly and remarkably promote cooperation among individuals, and interestingly, there exists an optimal range of the parameter of cautiousness leading to the highest level of cooperation. Moreover, we also investigate the problem regarding the initial conditions comprising different fractions of cooperators, and find that our results are very robust with respect to the initial conditions.

\section{The model}

We employ the prisoner's dilemma game (PDG) to model game interactions between individuals. The evolution of the game is taking place on a $N=L \times L$ square lattice with periodic boundary conditions where each site is occupied by an individual. The evolutionary process is iterated in accordance with the simulation procedure comprising the following interaction and strategy updating stages:

In the interaction stage, each individual performs PDG interactions with all immediate neighbors in the von Neumann neighborhood. The overall payoff of an individual is the accumulation of payoffs over all interactions. Following previous studies $[7,10]$, each individual calculates its payoff according to the following payoff values: $T=b, R=1$, and $P=S=0$, where $b$ is typically constrained to the interval $1.0 \leq b \leq 2.0$. Alternatively, another set of payoff parameters, i.e., $T=b, R=b-c, S=-c$ and $P=0$ can also be adopted, where $c$ is the cost an individual incurs to provide a benefit $b$ to the opponent [56]. Extensive simulations have confirmed that the following results are robust 
with respect to the changes in the payoff formulation.

After the interactions, each individual has an overall payoff obtained with the current strategy, and then the evolution of the game enters the updating stage. At this stage, each individual is allowed to update its strategy by learning a randomly chosen individual among immediate neighbors. For example, individual $i(0 \leq i<N)$ imitates the randomly chosen neighbor $j$. The probability for individual $i$ to imitate individual $j$ is

$$
W_{j \rightarrow i}=\frac{1}{1+\exp \left[\left(P_{i}-P_{j}+\sigma\right) / \kappa\right]}
$$

where $\kappa$ characterizes the noise introduced to permit irrational choices, and $P_{i}$ $\left(P_{j}\right)$ denotes the payoff of individual $i(j)$. Moreover, $\sigma$ is a dimensionless real number called cautiousness index, indicating the extent of the cautiousness of individuals, with values ranging from 0 to $+\infty$. Here for the simplicity but without loss of generality, we assume that all individuals have the same index of cautiousness. For $\sigma=0$, the traditional model where individuals have no cautiousness is recovered such that they begin to update their strategy as long as the payoff difference $P_{j}-P_{i}>0$ [9]. For $\sigma \rightarrow+\infty$, individuals have the highest level of cautiousness and will never change their strategy in the evolution.

The simulation of the evolution is performed with a synchronous updating rule on a $L=100$ lattice. We have verified that the presented results are robust to variations of the system size, as well as to the variation of the simulation procedure (e.g., by using random sequential updating rather than synchronous updating). The evolution of the system can reach a dynamical equilibrium after a suitable transient time $(t<5000)$, and then the density of cooperators reaches its asymptotic value and remains there within small fluctuations. We take this asymptotic value to describe the cooperation level in the whole population. The initial condition is also crucial for the evolutionary outcomes. Here we first consider the commonly used setup for the initial condition, that is, initially, $50 \%$ of the individuals are cooperators and $50 \%$ are defectors, who are randomly distributed over the square lattice. Then, we study the effect of different initial fractions of cooperators on the equilibrium frequency of cooperators. The key quantity for characterizing the system is the density of cooperators, which is defined as the fraction of cooperators in the whole population. 


\section{Results}

We first study the variation of the density of cooperators $\rho_{C}$ with the parameter $\sigma$ for different values of $\kappa$, as shown in Fig.1. The simulation results (left panel) are complemented by the pair approximation predications (right panel) $[57,58]$. One can observe from the simulation results that $\rho_{C}$ changes non-monotonically with increasing $\sigma$, irrespective of the value of $\kappa$. In particular, there exists a range of $\sigma$ (i.e., $0.3<\sigma \leq 0.8$ ) resulting in the highest cooperation level $\left(\rho_{C} \approx 0.82\right.$ ). As $\sigma \rightarrow \infty, \rho_{C}$ approaches 0.5 . This is because as $\sigma \rightarrow \infty$, no individuals will imitate their neighbors and therefore the initial density of cooperators is maintained. By comparing the results for $\sigma=0$ and $\sigma>0$, one can clearly find the remarkable promotion of cooperation. Varying the values of $\kappa$ can also have an effect on $\rho_{C}$. Specifically, in most of the range of $\sigma$ increasing $\kappa$ leads to the decline of $\rho_{C}$, although at moderate values of $\sigma$ cooperation can also be comparatively promoted. The most pronounced phenomenon should be the discontinuous phase transitions of $\rho$ with $\sigma$ for $\kappa=0$. For $\kappa>0$, because of the introduction of the stochasticity, the phase transitions disappear. From the right panel, one can observe that the pair approximation predications qualitatively agree with the simulation results, especially for $\sigma<1.5$, where the changing tendency of the simulation results is exactly captured by pair approximation, despite of the difference in the cooperation levels. Furthermore, the phase transitions are recovered, and all the phase transition points are well reproduced, which are $0.1,0.2,0.3,0.8$, $0.9,1.1,1.2,1.3,1.4,1.9,2.2,2.3$ and 2.4 , respectively.
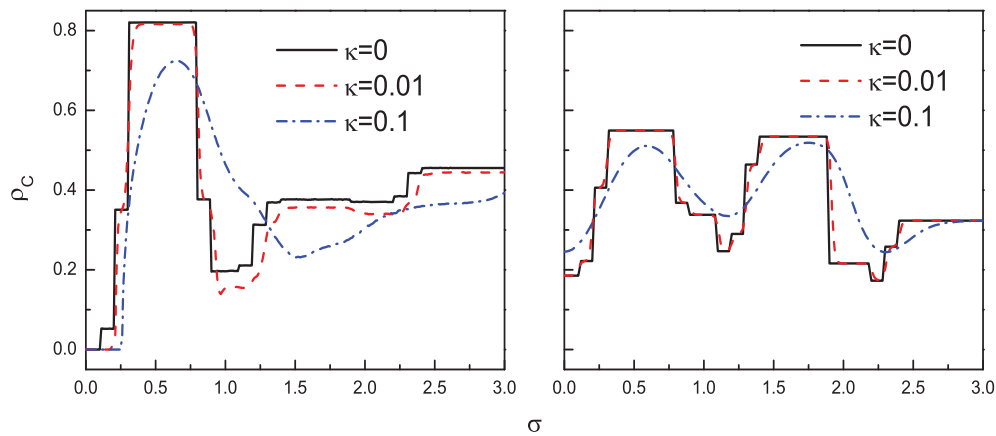

Fig. 1. Density of cooperators $\rho_{C}$ as a function of the parameter $\sigma$ for different values of $\kappa$. The left panel shows the simulation results and the right panel the pair approximation predications and both panels have the same vertical coordinate scale. The simulation results are obtained by averaging over 100 different realizations, and for each realization, we average over 5000 time steps after 10000 time steps. The simulation results are obtained for $b=1.1$.

In Fig.2, we show the density of cooperators $\rho_{C}$ as a function of the temptation to defect $b$ for different values of $\kappa$. One can find that, for the simulation results, overall $\rho_{C}$ changes non-monotonically with increasing $b$, and in partic- 
ular, for small $\kappa$ (e.g., $\kappa=0$ and $\kappa=0.01)$, high cooperation levels $\left(\rho_{C}>0.8\right)$ can maintain up to $b=1.2$, which is in sharp contrast with the result associated with $\sigma=0$, for which cooperators die out when $b$ is in the very close vicinity of $b=1.0$. For $\kappa=0$, phase transitions occur as well with increasing $b$. For $\kappa>0$, the variation of $\rho_{C}$ becomes smooth at the phase transition point due to the introduction of noises. From the right panel which shows the pair approximation predications, one can find that $\rho_{C}$ descends monotonically with $b$. Thus, the pair approximation predications can only characterize the variation tendency of the simulation results for small values of $b$. Despite this, the phase transitions and the phase transition points are well recovered, which are $1.2,1.3,1.4,1.6$, and 1.8 , respectively.
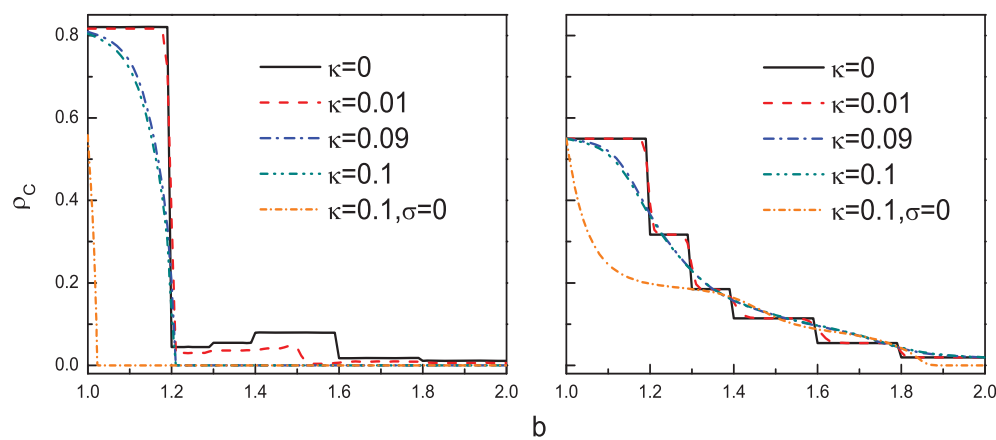

Fig. 2. Density of cooperators $\rho_{C}$ as a function of the temptation to defect $b$ for different values of $\kappa$. The left panel shows the simulation results and the right panel the pair approximation predications and both panels have the same vertical coordinate scale. The simulation results are obtained in the same way as in Fig.1. Here the parameter $\sigma$ is set to $\sigma=0.6$.

To demonstrate the effect of $b$ and $\sigma$ on cooperation comprehensively, we present a contour plot of the density of cooperators for $1.0 \leq b \leq 2.0$ and $0 \leq \sigma \leq 3.0$, as shown in Fig.3. Since the results for $\kappa=0$ are typical and the results for other values of $\kappa$ can be deduced from those for $\kappa=$ 0 , we present the results for $\kappa=0$. From the simulation results, one can find that the density of cooperators changes along certain lines (or, more generally, hyperplanes), which indicates the occurrence of phase transitions. Additionally, the pair approximation predications can overall characterize the profile of the simulation results, particularly for small values of $b$ and $\sigma$.

It remains to explain why individual cautiousness can promote cooperation in the prisoner's dilemma game. Before presenting the reason for the promotion of cooperation, let us first look at the typical snapshots for different values of $\sigma$, as shown in Fig.4. One can find that each value of $\sigma$ has its representative stationary pattern. Now we explain the promotion of cooperation induced by individual cautiousness. Assume that the imitation behavior occurs between a cooperator and a defector, as shown in Fig.5. Let us further assume that the cooperator has $n_{1}\left(0 \leq n_{1} \leq 3\right)$ cooperative neighbors and $n_{2}\left(1 \leq n_{2} \leq 4\right)$ 


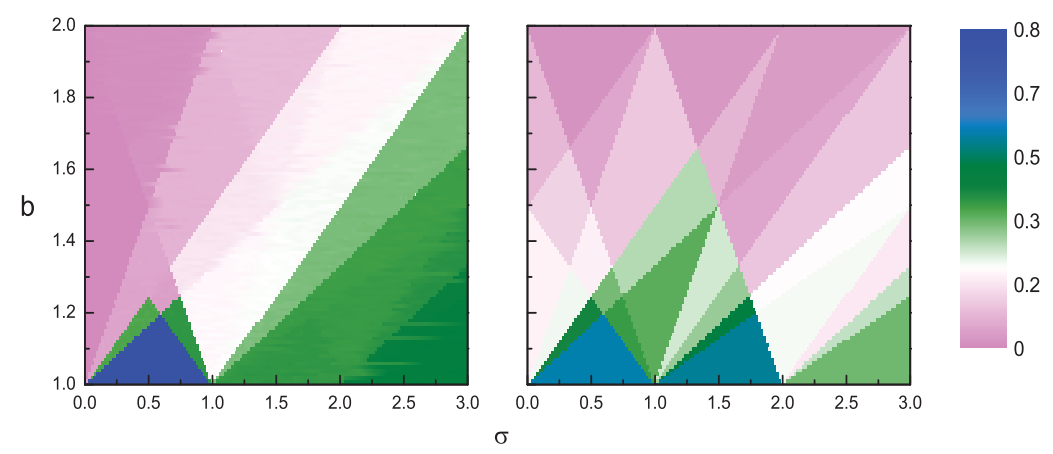

Fig. 3. Dependence of the density of cooperators on $b$ and $\sigma$. The left panel shows the simulation results and the right panel the pair approximation predications and both panels have the same vertical coordinate scale. The simulation results are obtained in the same way as in Fig.1. The depicted results are obtained for $\kappa=0$.

defecting neighbors $\left(n_{1}+n_{2}=4\right)$, and the defector has $n_{3}\left(1 \leq n_{3} \leq 4\right)$ cooperative neighbors and $n_{4}\left(0 \leq n_{4} \leq 3\right)$ defecting neighbors $\left(n_{3}+n_{4}=4\right)$. The payoffs of the cooperator and the defector are $n_{1} R+n_{2} S$ and $n_{3} T+$ $n_{4} P$, respectively. When individual cautiousness is considered, the imitation between the cooperator and the defector can be hampered when their payoff difference is smaller than $\sigma$, i.e., $\left|\left(n_{1} R+n_{2} S\right)-\left(n_{3} T+n_{4} P\right)\right|<\sigma$, which is responsible for the promotion of cooperation. For example, when $n_{1}=1$, $n_{2}=3, n_{3}=1$, and $n_{4}=3$, it is the case of $\sigma=0.15$ in Fig.4 (left panel) where two cooperators adjacent to each other are surrounded by defectors. In this scenario, the payoff of the cooperator is 1.0 and that of the defector is $b=1.1$. Therefore, when $\sigma=0.15$, since the payoff difference is smaller than the cautiousness index, the cooperators do not imitate their neighboring defectors such that the cooperators have the chance to survive. The rationale is similar for other values of $\sigma$ (see Fig.4).
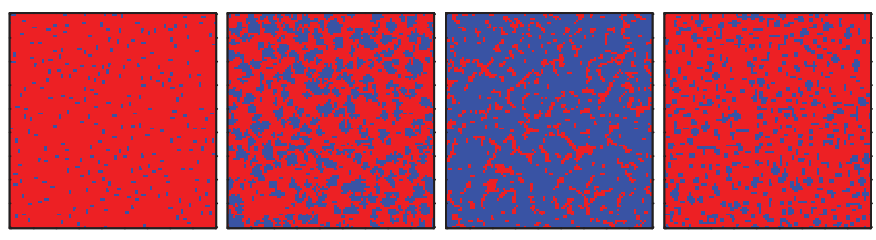

Fig. 4. Typical snapshots of the spatial patterns of cooperators (blue) and defectors (red) for $L=100, \kappa=0, b=1.1$ and $\sigma=0.15$ (left), $\sigma=0.25$ (middle left), $\sigma=0.5$ (middle right), and $\sigma=1.0$ (right) in the stationary state.

From Figs. 1, 2, and 3, one can observe that the level of cooperation changes along certain lines (or hyperplanes) where phase transitions occur with the variations of $b$ and $\sigma$. The emerging phase transitions can be explained by configuration analysis as above. Actually, the hyperplanes are all of the linear form $n_{1} R+n_{2} S=n_{3} T+n_{4} P-\sigma$ and $n_{1} R+n_{2} S-\sigma=n_{3} T+n_{4} P$. According to 
the strategy updating rule, for $\kappa=0$, the cooperator will imitate the defector if $n_{1} R+n_{2} S<n_{3} T+n_{4} P-\sigma$. Similarly, when $n_{1} R+n_{2} S-\sigma>n_{3} T+n_{4} P$, the defector will learn the cooperator. Given that $T=b, R=1$, and $P=$ $S=0$, the above two inequalities can be simplified as $n_{1}<n_{3} b-\sigma$ and $n_{1}-\sigma>n_{3} b$, respectively. Thus, the phase transition points can be obtained by $b=\left(n_{1}+\sigma\right) / n_{3}$ and $b=\left(n_{1}-\sigma\right) / n_{3}$. Given all possible combinations of $n_{1}$, $n_{2}, n_{3}$, and $n_{4}$, we can obtain all the appropriate values of $\sigma$ and $b$ at which phase transitions occur. According to our analysis, $\sigma=n_{3} b-n_{1}$. Thus, for $b=1.1$, the phase transition points can be $0.1,0.2,0.3,1.1,1.2,1.3,1.4,2.2$, 2.3 and 2.4. Similarly, from $\sigma=n_{1}-n_{3} b$, we can obtain the transition points 0.8, 0.9 and 1.9. Thus, one can find from Fig.1 that all the phase transition points exactly are predicted.

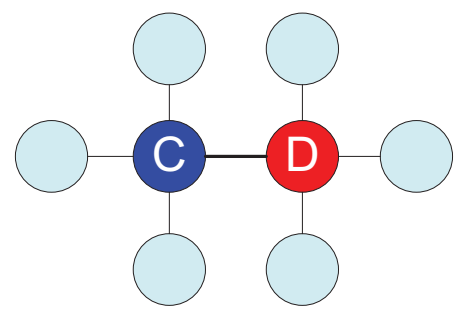

Fig. 5. Illustration of the imitation between a cooperator and a defector. The unspecified individuals can be either a cooperator or a defector.

Given that the initial condition regarding the proportion and distribution of cooperators and defectors on the square lattice plays an important role in the evolution of cooperation [47,59-61], we also studied the dependence of the equilibrium frequency of cooperators on random initial states with different fractions of cooperators $f_{I C}$ (note that $f_{I C}=0.5$ in most of previous studies). The density of cooperators $\rho_{C}$ as a function of $f_{I C}$ is shown in Fig.6. One can find that the density of cooperators saturates at small values of $f_{I C}$. For example, for $L=100, \rho_{C}$ increases monotonically with $f_{I C}$, and approximately saturates at $f_{I C}=0.1$. Note also that for different $L, \rho_{C}$ increases to the saturation along different trajectories with increasing $f_{I C}$. Specifically, the larger the population size, the less the initial cooperators are required for the saturation of the density of cooperators. That is, for the same $f_{I C}$, larger population sizes lead to a higher cooperation level when $f_{I C}$ is small (see Fig.6).

In order to expound the reason for the phenomenon above, we investigate the time evolution of the typical snapshots for $L=100$, as shown in Fig.7. One can find that, first, several very small patches of cooperators are established, and then larger cooperator clusters can be formed around these "nucleuses". Eventually, cooperators dominate the entire population. Actually, a small $2 \times 2$ 


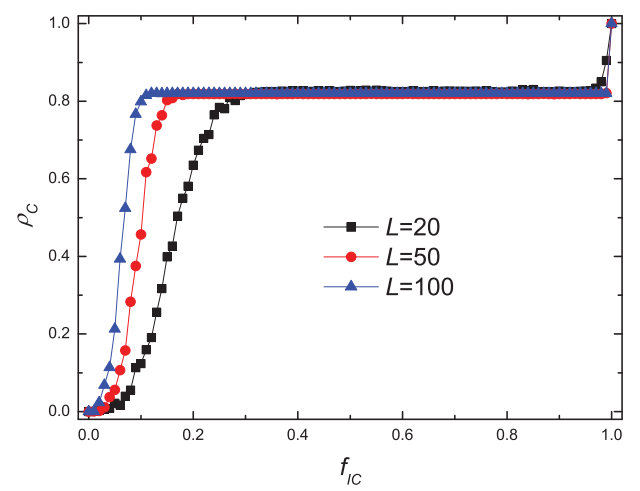

Fig. 6. Density of cooperators $\rho_{C}$ as a function of the initial fraction of cooperators $f_{I C}$ for different values of $L$. The results are obtained in the same way as in Fig.1. Depicted results are obtained for $b=1.1, \kappa=0$, and $\sigma=0.5$.

cluster of cooperators is enough for cooperators to flourish, as shown in Fig.8. One can find that originating from a $2 \times 2$ cooperator cluster, the evolution leads to the flourish of cooperators, reflected by the contiguous cluster with embedded strands of defectors (right panel of Fig.8). According to the present strategy updating rule, for $b=1.1$ and $\sigma=0.5$, as long as cooperators can simultaneously invade defectors at least two neighboring defectors, cooperators can eventually dominate the population (see the middle right and the right panels in Fig.9). The above fact indicates that the current results are very robust with respect to the initial condition.
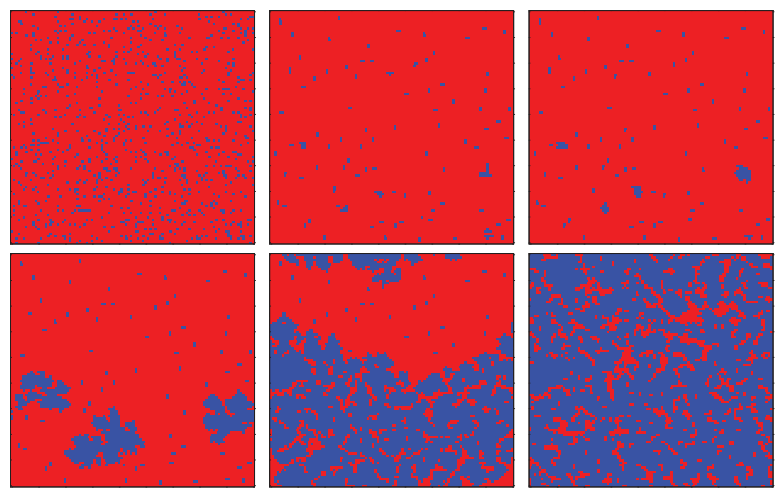

Fig. 7. Typical snapshots of the spatial patterns of cooperators (blue) and defectors (red) in the evolutionary process for $L=100, \kappa=0, b=1.1, \sigma=0.5$, and $f_{I C}=0.1$. The snapshots are taken at $t=0$ (top left), $t=10$ (top middle), $t=20$ (top right), $t=100$ (bottom left), $t=300$ (bottom middle), and $t=500$ (bottom right), respectively.

Now we can explain why for the same fraction of the initial cooperators, cooperation is more likely to emerge and thrive for larger population sizes. This is because for the same value of $f_{I C}$, when the population size is large, the number of the initial cooperators is larger such that the chance for cooperators to form small clusters (e.g., a square of four cooperators) initially is higher. As long as such "seeds" for the dominance of cooperation are formed, cooperation 
can thrive. Therefore, cooperation rises more quickly for larger population sizes.
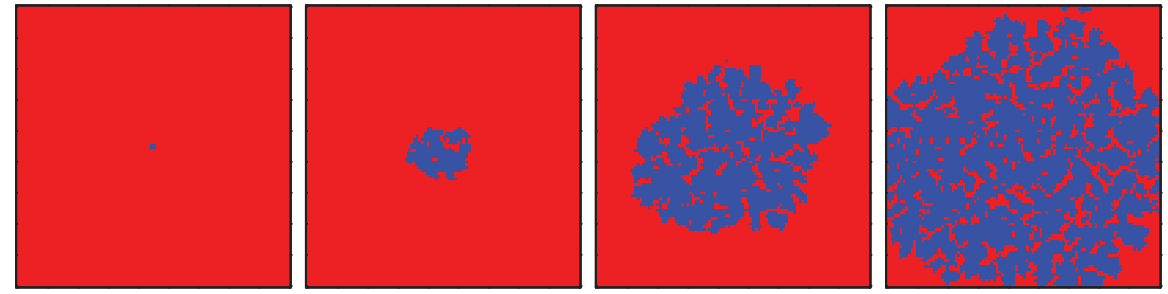

Fig. 8. Typical snapshots of the invasion of cooperators (blue) in a world of defectors (red) for $L=100, \kappa=0, b=1.1$, and $\sigma=0.5$. The snapshots are taken at $t=0$ (left), $t=100$ (middle left), $t=300$ (middle right), and $t=500$ (right), respectively.
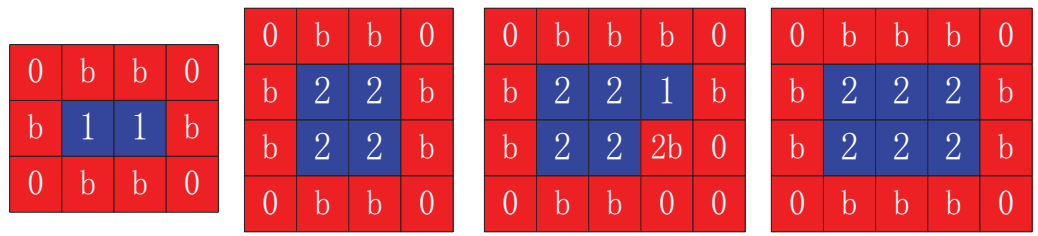

Fig. 9. Illustrations of the typical initial configurations of cooperators (blue) and defectors (red). Each small square corresponds to a single player. Denoted values correspond to the payoffs of individual players, as obtained for the presented configurations.

\section{Discussion and conclusion}

In summary, we have studied the impact of individual cautious strategy update on the evolution of cooperation in the spatial prisoner's dilemma game. It is found that when the cautiousness of individuals is introduced, cooperation can be remarkably promoted. Especially for small values of the cautiousness parameter, e.g., $0.3<\sigma<0.8$, the dominance of cooperators can be achieved. This is in sharp contrast to the results obtained with no individual cautiousness, where the traditional version of the spatial prisoner's dilemma game is essentially recovered. The observed promotion of cooperation has been explained by configuration analysis. The promotion of cooperation can be attributed to the equilibrium between cooperators and defectors induced by individual cautiousness. Specifically, when individual cautiousness is introduced, cooperators can survive when the advantage of defectors over cooperators is smaller than the parameter characterizing the cautiousness of individuals. Thus, cooperators win the chance to subsist and even invade defectors. We have also investigated the sensitivity of the density of cooperators to the initial states 
consisting of different fractions of cooperators. It is found that cooperation is very robust with respect to the initial condition reflected by the fact that a small faction of cooperators can lead to the dominance of cooperators. For the same unfavorable initial condition, larger lattice sizes are more beneficial to cooperation. We have also employed the pair approximation method to support our simulation results with semi-analytical calculations and to explain the observed transitions to different levels of cooperation on the square lattice.

Previous studies have already shown that individual characteristics can profoundly affect the evolution of cooperation in evolutionary games. Here we show that cautiousness as an important individual characteristic can favor the emergence of cooperation. The concept of cautiousness has been proposed in Ref.[62], which is referred to as the cost of strategy change. Since with increasing $\sigma$, the strategy updating behavior is increasing inhibited, which reflects the cautiousness or hesitation of individuals in strategy update in the real world, we therefore call it the index of individual cautiousness. However, Ref.[62] primarily investigated the role of the voluntary participation in the public goods game instead of the so-called "cost of strategy change". In Ref.[63], the authors proposed the concept of inertia and investigate its effect on the evolution of cooperation. The inertia there is similar to the cautiousness here in the sense that they both can control and regulate the learning activity of individuals. However, they are different in the controlling way of the learning activity. In Ref.[63], the inertia is an artificially created index which is closely correlated with individual number of neighbors. While here the cautiousness is concerned with the payoff of individuals and has the same dimension as payoff. Given that individuals are usually sensitive to the payoff, the cautiousness here is of great practical significance.

Moreover, unlike in the majority of previous works, in our case the strategy update is not unconditional but depends on individual cautiousness. If the payoff difference between an individual and the selected neighbor is higher than the index of individual cautiousness, the imitation of the individual towards its neighbor occurs. Otherwise, nothing happens, that is, individuals try to update their strategy only if the strategy update is indeed profitable. While in most of previous works, the strategy update is reckless to some extent, since individuals update their strategy as long as the neighbors perform better. In Refs.[46,47], Liu et al. investigated the aspiration-based conditional learning, which is similar to the present work in the sense that the strategy updates are both conditional. On the other hand, the introduction of individual cautiousness can result in different time scales of individuals between interactions and strategy update [35-38]. We hope that this study will enrich our knowledge on how to successfully resolve the prisoner's dilemma, and is also helpful in understanding the ubiquitous cooperative behavior in the real world where the conflict of individual and collective interests widely exists. Our work may also have potential implications in designing coordination and 
cooperation mechanisms in multi-agent systems.

\section{Acknowledegments}

Y. Liu gratefully acknowledges the supported from Special Fund for the Basic Scientific Research of Central Colleges, Chang'an University under grant No. CHD2010JC134, the support from Special Fund for the Basic Research Support Planning of Chang'an University, the support from the open fund of road traffic intelligent detection and equipment engineering research center, Shaanxi province. L. Zhang, L. Ren and L. Wang gratefully acknowledge the support from National Natural Science Foundation of China (NSFC) under grant Nos. 61074144, 61103096 and 10972002, respectively.

\section{Appendix: Pair-approximation}

According to the pair approximation method [57,58], the rate equations of cooperator-cooperator $(\mathrm{c}, \mathrm{c})$ and cooperator-defector $(\mathrm{c}, \mathrm{d})$ edges are as follows:

$$
\begin{aligned}
\dot{p}_{c, c}= & \sum_{x, y, z}\left[n_{c}(x, y, z)+1\right] p_{d, x} p_{d, y} p_{d, z} \times \\
& \sum_{u, v, w} p_{c, u} p_{c, v} p_{c, w} f\left[P_{d}(x, y, z), P_{c}(u, v, w)\right] \\
& -\sum_{x, y, z} n_{c}(x, y, z) p_{c, x} p_{c, y} p_{c, z} \times \\
& \sum_{u, v, w} p_{d, u} p_{d, v} p_{d, w} f\left[P_{c}(x, y, z), P_{d}(u, v, w)\right] \\
\dot{p}_{c, d}= & \sum_{x, y, z}\left[1-n_{c}(x, y, z)\right] p_{d, x} p_{d, y} p_{d, z} \times \\
& \sum_{u, v, w} p_{c, u} p_{c, v} p_{c, w} f\left[P_{d}(x, y, z), P_{c}(u, v, w)\right] \\
& -\sum_{x, y, z}\left[2-n_{c}(x, y, z)\right] p_{c, x} p_{c, y} p_{c, z} \times \\
& \sum_{u, v, w} p_{d, u} p_{d, v} p_{d, w} f\left[P_{c}(x, y, z), P_{d}(u, v, w)\right]
\end{aligned}
$$

where $\mathrm{x}, \mathrm{y}, \mathrm{z}$ each are either a cooperator or a defector and $n_{c}(x, y, z)$ denotes the number of cooperators among them. Moreover,

$$
f\left(P_{i}, P_{j}\right)=\frac{1}{1+\exp \left[\left(P_{i}-P_{j}+\sigma\right) / \kappa\right]}
$$


where $P_{i}$ and $P_{j}$ are the payoffs of the two neighboring individuals $i$ and $j$, $\sigma$ denotes the index of the cautiousness, and $\kappa$ represents the intensity of selection. By performing numerical integration for the above two differential equations and in combination with the symmetry condition $p_{c, d}=p_{d, c}$, as well as the constraint $p_{c, c}+p_{c, d}+p_{d, c}+p_{d, d}=1$, we can obtain $\rho_{C}=p_{c, c}+p_{c, d}$.

\section{References}

[1] R. Axelrod, The Evolution of Cooperation, Basic Books, New York, 1984.

[2] M.A. Nowak, Evolutionary Dynamics: Exploring the Equations of Life, Harvard University Press, Cambridge MA, 2006.

[3] J. Maynard Smith, Evolution and the Theory of Games, Cambridge University Press, Cambridge, 1982.

[4] K. Sigmund, Games of Life: Exploration in Ecology, Evolution and Behavior, Oxford University Press, Oxford UK, 1993.

[5] J. Hofbauer, K. Sigmund, Evolutionary Games and Population Dynamics, Cambridge University Press, Cambridge England, 1998.

[6] R.M. Dawes, Annu. Rev. Psychol. 31 (1980) 169.

[7] M.A. Nowak, R.M. May, Nature 359 (1992) 826.

[8] G. Szabó, C. Fáth, Phys. Rep. 446 (2007) 97.

[9] G. Szabó, C. Töke, Phys. Rev. E 58 (1998) 69.

[10] F.C. Santos, J.M. Pacheco, Phys. Rev. Lett. 95 (2005) 098104.

[11] J. Vukov, G. Szabó, Phys. Rev. E 71 (2005) 036133.

[12] F.C. Santos, J.M. Pacheco, T. Lenaerts, Proc. Natl. Acad. Sci. USA 103 (2006) 3490 .

[13] F.C. Santos, J.F Rodrigues, J.M. Pacheco, Proc. R. Soc. B 273 (2006) 51.

[14] J. Gómez-Gardeñes, M. Campillo, Y. Moreno, L.M. Floría, Phys. Rev. Lett. 98 (2007) 108103.

[15] X. Chen, F. Fu, L. Wang, Physica A 378 (2007) 512.

[16] F. Fu, L.-H. Liu, L. Wang, Eur Phys J B 56 (2007) 367.

[17] Y.-K. Liu, Z. Li, X.-J. Chen, L. Wang, Chin. Phys. B 18 (2009) 2623.

[18] Y.-K. Liu, Z. Li, X.-J. Chen, L. Wang, Chin. Phys. Lett. 26 (2009) 048902.

[19] M. Perc, A. Szolnoki, BioSystems 99 (2010) 109. 
[20] F. Fu, X. Chen, L. Liu, L. Wang, Physica A 383 (2007) 651.

[21] F. Fu, L. Wang, Phys. Rev. E 78 (2008) 016104.

[22] F. Fu, T. Wu, L. Wang, Phys. Rev. E 79 (2009) 036101.

[23] S. Van Segbroeck, F.C. Santos, T. Lenaerts, J.M. Pacheco, Phys. Rev. Lett. 102 (2009) 058105.

[24] Y. Liu, Z. Li, X. Chen, L. Wang, Physica A 390 (2011) 43.

[25] G. Szabó, J. Vukov, A. Szolnoki, Phys. Rev. E 72 (2005) 047107.

[26] M. Perc, New J Phys 8 (2006) 22.

[27] J. Vukov, G. Szabó, A. Szolnoki, Phys. Rev. E 73 (2006) 067103.

[28] A. Szolnoki, J. Vukov, G. Szabó, Phys. Rev. E 80 (2009) 056112.

[29] G. Szabó, A. Szolnoki, J. Vukov, EPL 87 (2009) 18007.

[30] H. Ohtsuki, M.A. Nowak, J.M. Pacheco, Phys. Rev. Lett. 98 (2007) 108106.

[31] H. Ohtsuki, J.M. Pacheco, M.A. Nowak, J. Theor. Biol. 246 (2007) 681.

[32] X. Chen, F. Fu, L. Wang, Phys. Rev. E 78 (2008) 051120.

[33] M. Perc, A. Szolnoki, Phys. Rev. E 77 (2008) 011904.

[34] F.C. Santos, M.D. Santos, J.M. Pacheco, Nature 454 (2008) 213.

[35] Z.-X. Wu, Z. Rong, P. Holme, Phys. Rev. E 80 (2009) 036103.

[36] C.P. Roca, J.A. Cuesta, A. Sánchez, Phys. Rev. Lett. 97 (2006) 158701.

[37] A. Szolnoki, M. Perc, Z. Danku, EPL 84 (2008) 50007.

[38] Z. Rong, Z.-X. Wu, W.-X. Wang, Phys. Rev. E 82 (2010) 026101.

[39] Y. Liu, Z. Li, X. Chen, L. Wang, Physica A 389 (2010) 2390.

[40] A. Szolnoki, G. Szabó, EPL 77 (2007) 30004.

[41] A. Szolnoki, M. Perc, New J. Phys. 10 (2008) 043036.

[42] G. Szabó, A. Szolnoki, Phys. Rev. E 79 (2009) 016106.

[43] J.-Y. Guan, Z.-X. Wu, Z.-G. Huang, X.-J. Xu and Y.-H Wang, Europhys. Lett. 76 (2006) 1214.

[44] Z.-X. Wu, X.-J. Xu, Z.-G. Huang, S.-J Wang, Y.-H. Wang, Phys. Rev. E 74 (2006) 021107.

[45] X. Chen, L. Wang, Phys. Rev. E 77 (2008) 017103.

[46] Y. Liu, X. Chen, L. Wang, B. Li, W. Zhang, H. Wang, EPL 94 (2011) 60002.

[47] Y. Liu, X. Chen, L. Zhang, L. Wang, M. Perc, PLoS ONE 7 (2012) e30689. 
[48] D. Helbing, W.-J. Yu, Proc. Natl. Acad. Sci. USA 106 (2009) 3680.

[49] W.-J. Yu, Phys. Rev. E 83 (2011) 026105.

[50] T. Wu, F. Fu, L. Wang, PLoS ONE 6 (2011) e27669.

[51] T. Wu, F. Fu, Y. Zhang, L. Wang, Phys Rev E 85 (2012) 066104.

[52] X. Chen, F. Fu, L. Wang, Physica A 387 (2008) 5609.

[53] Y.-K. Liu, Z. Li, X.-J. Chen, L. Wang, Chin. Phys. Lett. 26 (2009) 088902.

[54] X. Chen, L. Wang, Phys. Rev. E 80 (2009) 046109.

[55] X. Chen, F. Fu, L. Wang, Phys. Rev. E 80 (2009) 051104.

[56] M.A. Nowak, Science 314 (2006) 1560.

[57] C. Hauert, M. Doebeli, Nature 428 (2004) 643.

[58] C. Hauert, G. Szabó, Game theory and physics, Am J Phys 73 (2005) 405.

[59] X. Chen, F. Fu, L. Wang, Phys. Lett. A 372 (2008) 1161.

[60] F. Fu, M.A. Nowak, C. Hauert, J. Theor. Biol. 266 (2010) 358.

[61] P.-P Li, J. Ke, Z. Lin, P.M. Hui, Phys. Rev. E 85 (2012) 021111.

[62] G. Szabó, C. Hauert, Phys. Rev. Lett. 89 (2002) 118101.

[63] R.-R. Liu, Z. Rong, C.-X Jia, B.-H. Wang, EPL 91 (2010) 20002. 